\title{
ALIGNMENT OF CLUSTERS WITH BRIGHTEST MEMBER GALAXIES
}

Gregory S. Tucker and J. B. Peterson

Joseph Henry Labs., Princeton University, Princeton, N.J. 08544 U.S.A.

We have obtained CCD images of the brightest elliptical galaxy in 27 clusters of galaxies with redshifts $z<0.1$. For each galaxy we fitted elliptical isophotes to the galaxy image. We also measured the position angle of each cluster from the Palomar Observatory Sky Survey (POSS) prints. We find only marginal evidence for alignment of brightest cluster members (BCM) with their parent clusters, but we find that our CCD isophotes are well aligned with the POSS image.

Argyres, Groth, Peebles, and Stuble (AGPS) have detected alignment of the POSS image of BCM gal axies with the Shane-Wirtanen counts of galaxies around the cluster. The anisotropy is small (10\%) but significant out to $4^{\circ}$. We find that the $-6^{\prime \prime}$ isophotes of BCM galaxies are well aligned with the POSS image, on average the angles differ by $13^{\circ}$. If this alignment is present throughout the AGPS sample alignment of structure extends from $4 h^{-1} \mathrm{kpc}$ to $15 \mathrm{~h}^{-1} \mathrm{Mpc}$.

This research was supported by a grant from the National Science Foundation. 